\title{
DESEMPENHO DE ESCOLARES DE 1ª SÉRIE NA BATERIA DE IDENTIFICAÇÃO DE ERROS DE REVERSÃO E INVERSÃO NA ESCRITA: ESTUDO PRELIMINAR
}

\section{Performance of students from $1^{\text {st }}$ grade in the inversion and reversion errors in the written identification test: preliminary study}

\author{
Beatriz Aparecida Gimenez Gonçalves ${ }^{(1)}$, Simone Aparecida Capellini (2)
}

\begin{abstract}
RESUMO
Objetivo: caracterizar o desempenho de escolares da $1^{a}$ série na bateria de identificação de erros de reversão e inversão na escrita. Métodos: participaram deste estudo 30 escolares de $1^{\underline{a}}$ série de ensino público do município da cidade de Marília-SP, de ambos os gêneros, na faixa etária de 7 anos a 7 anos e 11 meses de idade. Como procedimento foi realizada a adaptação brasileira da bateria de identificação dos erros de reversão e inversão na escrita. Esta bateria é composta por 3 testes: teste de desempenho contínuo, teste de memória de curto prazo e teste de controle de escrita. Cada teste é composto por subtestes que visam identificar a capacidade dos escolares em identificar as inversões e reversões de letras e números isolados e letras e números em sequências. Resultados: os resultados revelaram que os escolares da $1^{\underline{a}}$ série apresentaram desempenho superior para identificação de reversão e inversão de letras dentro de palavras do que isoladamente, identificação da reversão para letras e números com desempenho superior se comparado a inversão de letras na palavra. Além disso, os escolares deste estudo apresentaram menor tempo para identificação de reversão e inversão de letras e números isolados do que em sequência. Conclusão: a Bateria adaptada para este estudo permitiu conhecer o perfil dos escolares de 1aㅡ série quanto a identificação dos erros de inversão e reversão, entretanto, é necessário novas aplicações para verificar a ocorrência de identificação destes erros em populações de diferentes fase de alfabetização.
\end{abstract}

DESCRITORES: Avaliação; Escrita Manual; Aprendizagem

\section{INTRODUÇÃO}

O período da alfabetização é o momento em que há uma superposição de habilidades para a ocorrência da aprendizagem da leitura e escrita. Essa aprendizagem envolve habilidades cognitivas, linguísticas e motoras que exigem dos escolares o uso dos componentes sensório-motores e perceptivos, ou seja, a capacidade de decodificação

(1) Aluna do Curso de Graduação em Fonoaudiologia da Faculdade de Filosofia e Ciências da Universidade Estadual Paulista, FFC/UNESP, Marília, SP.

(2) Fonoaudióloga; Docente do Departamento de Fonoaudiologia e do Programa de Pós-Graduação em Educação da Faculdade de Filosofia e Ciências da Universidade Estadual Paulista, FFC/UNESP, Marília, SP; Doutora em Ciências Médicas pela Universidade Estadual de Campinas.

Conflito de interesses: inexistente das palavras e a ação motora adequada para a execução do ato motor da escrita ${ }^{1}$.

Segundo a literatura ${ }^{2}$, o processo de aquisição da linguagem escrita, assim como o da linguagem oral, envolve diversas regiões cerebrais, entre elas a área parieto-occipital. Na região occipital, o córtex visual primário é o responsável pelo processamento dos símbolos gráficos, e as áreas do lobo parietal são responsáveis pelas questões visuo-espaciais da grafia. Essas informações processadas são reconhecidas e decodificadas na área de Werneck, responsável pela compreensão da linguagem, e a expressão da linguagem escrita necessita da ativação do córtex motor primário e da área de Broca. Para todo este processo ocorrer, é importante que as fibras de associação intra-hemisféricas estejam intactas. 
Na região anterior do cérebro ocorrem o planejamento, organização e execução do movimento. Outras áreas também participam da ação motora, enviando mensagens, dosando a força, a agilidade, fornecendo feedback visual, táctil e auditivo, permitindo, desta forma, o ajuste constante do movimento ${ }^{3}$. Na região frontal, o movimento acontece da seguinte forma: primeiramente, há uma intenção de movimento, um planejamento elaborado no córtex pré-frontal; em seguida, essa informação passa para a área pré-motora (que fica entre o lobo pré-frontal e a área motora), que é responsável por organizar a seqüência motora; posteriormente, esta é projetada na área motora primária (que fica no giro pericentral), que enviará os impulsos (via medula) para a musculatura, a fim de executar o movimento planejado ${ }^{3}$.

A existência de uma combinação de fenômenos biológicos e ambientais no aprendizado da linguagem escrita, envolvendo a integridade motora, a integridade sensório-perceptual e a integridade socioemocional (possibilidades reais que 0 meio oferece em termos de quantidade, qualidade e frequência de estímulos) já foram descritas na literatura ${ }^{4} \mathrm{e}$ atualmente assumem um papel essencial no entendimento do processamento fonológico e ortográfico para a realização da leitura e da escrita ${ }^{5}$.

Os estudos sobre a leitura são diversificados e aparecem sob os mais diversos contextos, tanto na literatura nacional ${ }^{6-8}$ como na literatura internacional ${ }^{9-12}$, abordando o processo de decodificação e compreensão da leitura ou os programas de remediação. Entretanto, o mesmo não ocorre com a escrita em âmbito nacional. Os estudos internacionais ${ }^{13,14}$ enfocam além de questões ortográficas, questões morfológicas e sintáticas, enquanto os estudos nacionais ${ }^{15,16}$ enfocam principalmente questões ortográficas.

O sistema de escrita do português brasileiro tem base alfabética e por isso para ser adquirido pela criança é necessária a formação e recuperação da palavra da memória, baseada na informação visual, fonológica e ortográfica. As tentativas de leitura e escrita por parte de leitores e escritores iniciantes têm, em geral, mais sucesso se estes compreenderem o que é o princípio alfabético, pois, dessa forma, os leitores poderão fazer uso, de maneira mais eficaz, da informação obtida pela decodificação letra-som ${ }^{17,18}$.

Para se chegar à descoberta do fonema, deve-se adquirir e desenvolver a consciência fonológica, que se trata da competência metalinguística que possibilita o acesso consciente ao nível fonológico da fala e à manipulação cognitiva das representações neste nível ${ }^{19-21}$. Porém, o sistema de escrita do português brasileiro não apresenta apenas a característica unívoca de relação letra-som, há, também, a correspondência múltipla do som na escrita, o que exige dos escolares memória visual, além do conhecimento e domínio de regras de correspondência grafofonêmicas que não dependem de uma conversão direta letra-som 17,20,22.

Estudos nacionais ${ }^{22,23}$ apontam para algumas questões ortográficas que são apropriadas pelas crianças de forma mais lenta, exigindo destas, maior esforço, como no caso dos erros por confusão entre as terminações am e ão e os erros relacionados à representação múltipla do fonema na grafia. Os erros de reversão e inversão são pouco descritos na literatura e quando isto ocorre, os estudos sugerem que estes erros podem não incorrer na população brasileira devido à ortografia do português apresentar maior transparência do que opacidade para a escrita em comparação com ortografia do inglês ${ }^{24}$.

Porém, os erros de inversão e reversão são descritos como presentes na população de crianças que apresentam dislexia ou distúrbio de aprendizagem, pois estas apresentam alterações de ordem perceptivo-viso-motora ${ }^{25}$. Entretanto, não há na literatura nacional estudos que utilizem protocolos específicos para a investigação dos erros de inversão e reversão e sua etiologia.

Com base no exposto, este estudo teve por objetivos caracterizar o desempenho de escolares da $1^{\text {a }}$ série na bateria de identificação de erros de reversão e inversão na escrita.

\section{MÉTODOS}

Este estudo se caracteriza por ser experimental e transversal, realizado com escolares do ensino público municipal da cidade de Marília-SP.

Participaram deste estudo 30 escolares da $1^{\text {a }}$ série do ensino fundamental municipal, de ambos os gêneros e na faixa etária de 7 anos a 7 anos e 11 meses, sendo $57 \%$ do gênero feminino e $43 \%$ do gênero masculino.

Os escolares que participaram deste estudo não apresentaram anotações referentes à deficiência mental, física, sensorial ou múltipla em prontuário escolar.

Para a realização deste estudo, foram aplicados os seguintes procedimentos:

- Termo de Consentimento Pós-Informado: Conforme resolução do Conselho Nacional de Saúde CNS 196/96, anteriormente ao início das avaliações, os pais ou responsáveis dos escolares assinaram o termo de Consentimento PósInformado, autorizando a participação de seu filho/filha no estudo. 
- Bateria de identificação dos erros de reversão na leitura e na escrita: Foi realizada a adaptação brasileira da Bateria de testes de identificação dos erros de reversão na leitura e na escrita ${ }^{26}$. A Bateria é composta por 3 testes divididos em subtestes conforme descritos a seguir:

1. Teste de desempenho contínuo: O objetivo deste teste foi verificar a habilidade dos participantes em identificar rapidamente letras, número e palavras com reversão e inversão. Os subtestes estão descritos abaixo:

1.1) Subteste de Controle de atenção 1: 0 escolar deveria sublinhar a letra-alvo (letra c) toda vez que aparecesse. Este subteste incluiu a mistura de letras maiúsculas e minúsculas das seguintes letras: B, E, F, G, R, A, D, H, I, J, Q, T, V, Y, a, c, e, $h, j, k, r, y, i, l, o, v$, que apareciam duas vezes na lista. A letra-alvo apareceu 14 vezes, resultando em 64 vezes de ocorrência de aparecimento. $O$ resultado foi analisado pelo número de acertos na identificação dos alvos.

1.2) Subteste de Controle de atenção 2: 0 escolar deveria sublinhar a letra-alvo (letra c) toda vez que aparecesse. Este subteste envolveu 40 sequências de 4 letras (ex: Bjkv), sendo que 10 delas apresentavam a letra alvo (c) nas quatro posições possíveis (ex: Glic). O resultado foi analisado pelo número de acertos na identificação dos alvos.

1.3) Detecção de reversão 1: O escolar deveria sublinhar o número que aparecesse de forma invertida (espelho). Este subteste incluiu 50 números (excluindo 6 e 9), onde os números 2, 3, 4, 5 e 7 apareceram invertidos (espelho - 2, 3, 4, 5 e 7) distribuídos duas vezes cada um, totalizando 10 alvos. Os outros números 1, 2, 3, 4, 5, 7 e 8 foram apresentados na forma convencional, sendo que 0 5 e 7 apareceram cinco vezes cada e o restante seis vezes cada, totalizando 40 itens. $O$ resultado foi analisado pelo número de acertos na identificação dos alvos.

1.4) Detecção de reversão 2: O escolar deveria sublinhar a letra que aparecesse de forma invertida ou revertida. Este subteste incluiu 65 letras misturadas, em que 13 letras são os alvos, pois apareciam invertidas (B, E, F, G, R, a, c, e, h, j, k, r, y). As letras não-alvos são $B, E, F, G, R, a, c, e, h, i, k$, r, y, A, D, H, I, J, Q, T, V, Y, i, I, o, v, e apareceram duas vezes de forma normal, totalizando 52 itens. $O$ resultado foi analisado pelo número de acertos na identificação dos alvos.

1.5) Detecção de reversão 3: O escolar deveria sublinhar a letra que aparecesse de forma invertida ou revertida. Este subteste envolveu 40 sequências de 4 letras (ex: Qryk), sendo que 10 delas são alvos, pois apresentavam uma letra modificada nas quatro posições possíveis (ex: rDGo). O resultado foi analisado pelo número de acertos na identificação dos alvos.

1.6) Palavras invertidas 1: O escolar deveria sublinhar a palavra que apresentasse uma letra de forma invertida ou revertida. Este subteste incluiu 80 palavras de 4 letras, em que 64 delas apareceram com ortografia convencional e as outras 16 são as alvos, apresentando alguma letra modificada. Foram apresentadas 8 palavras com as letras j, e, y, c, r, k, h, com reversão de espelho e outras 8 palavras com f, w, m, t, b, d, n, p invertidas $(m-w)$ ou visão de espelho $(b-d)$, que resultassem em outra letra. As mesmas letras apareciam nas palavras escritas de forma convencional e equilibrada. O resultado foi analisado pelo número de acertos na identificação dos alvos.

2. Teste de memória de curto prazo: Essa prova se diferencia da prova de desempenho contínuo, pois avalia o desempenho da memória a curto prazo, além de conhecimento prévio dos itens da amostra.

2.1) Detecção de reversão 4: O escolar deveria sublinhar a letra e número que aparecesse de forma invertida ou revertida. Este subteste incluiu 42 itens, em que 26 são letras (b, d, p, q, m, w, f, t, n, u, N, Z,B, E, F, G, J, K, L, R, a, c, e, h, r, y) e 6 números $(2,3,4,5,6,7)$, totalizando 32 itens não-alvos. Os itens alvos foram 7 das letras normais $(E, G, K, R, c$, $h, y)$ e 3 números $(3,5,7)$ que apareciam invertidos em forma de espelho, misturados com as letras não-alvo. O resultado foi analisado pelo número de acertos na identificação dos alvos.

2.2) Palavras invertidas 2: O escolar deveria sublinhar a palavra que apresentasse uma letra de forma invertida ou revertida. Este subteste incluiu 16 palavras de quatro letras, sendo 8 delas com letras invertidas, ocupando todas as posições possíveis, contrabalanceadas. Uma parte apresentava as letras $f, w, m, t, b, d, n, p$ e a outra apresentava as letras j, e, y, c, r, k, h, a. O resultado foi analisado pelo número de acertos na identificação das letras alvos.

3. Prova de controle da escrita: Essa prova foi desenvolvida para verificar a incidência de reversões quando as condições ortográficas eram favorecedoras, além das pistas visuais e auditivas diminuírem.

3.1) Escrita a partir de figuras: O escolar deveria escrever o que as figuras representam. Este subteste incluiu 18 figuras, na qual 10 figuras apresentavam uma letra que reverte $(n, m, b, f, p, w$, $\mathrm{t}, \mathrm{d}, \mathrm{u}, \mathrm{q})$ e as outras $8 \mathrm{com}$ palavras sem letras que revertem (c, j, e, g, r, a, s, h). O resultado foi analisado quando a produção apresentou alguma inversão ou reversão. 
3.2) Cópia: O escolar deveria copiar as letras exatamente da forma que lhe foi apresentado. Este subteste incluiu 44 letras, na qual 12 são casos de reversão misturados (b, d, p, q, n, u, m, w, f, t, N, Z), aparecendo uma vez cada e 16 normais $(B, D$, E, F, G, J, K, L, R, a, c, e, g, h, r, y), aparecendo duas vezes cada, uma vez normal e outra invertida (espelho). Quando a letra aparecesse na orientação normal, os escolares deveriam copiar a letra na sua forma normal, mas se a letra aparecesse invertida (espelho), os escolares deveriam copiar a letra desta forma. O resultado foi analisado quando a produção de escrita dos escolares apresentasse alguma inversão ou reversão.

O procedimento foi aplicado individualmente em uma única sessão de 60 minutos realizada em período de aula na escola de realização do estudo.

Este trabalho foi aprovado pelo Comitê de Ética em Pesquisa da Universidade Estadual Paulista CEP/FFC/UNESP/Marília - SP, sob o protocolo número 3350/2008.

Os resultados foram analisados estatisticamente utilizando o Teste dos Postos Sinalizados de Wilcoxon, com o intuito de verificar possíveis diferenças entre o escore esperado e o obtido nos subtestes da Bateria de identificação dos erros de reversão na leitura e na escrita e o Teste de Friedman, com o objetivo de verificar possíveis diferenças entre as variáveis da mesma prova.

Para a análise estatística, foi adotado o nível de significância de $5 \%(\alpha=0,050$ - significância adotada), marcado com asterisco. A análise dos dados foi realizada utilizando o programa SPSS (Statistical Package for Social Sciences), em sua versão 13.0.

\section{RESULTADOS}

Nesta seção serão apresentados os resultados em tabela, a qual além de apresentar a média, desvio padrão e valor de $p$, também apresentará os valores mínimos e máximos de cada prova, sendo que o valor mínimo é considerado como o efeito base e o valor máximo como efeito teto para cada subteste realizado pelos escolares.

A Tabela 1 apresenta o desempenho dos escolares de $1^{\text {a }}$ série na bateria de identificação dos erros de reversão na leitura e na escrita. Os dados apresentados são referentes ao desempenho esperado e obtido em cada prova.

Com a aplicação do Teste dos Postos Sinalizados de Wilcoxon para a comparação do desempenho esperado e obtido, verificou-se diferença estatisticamente significante em todos os testes da Bateria, evidenciando que os escolares de $1^{\text {a }}$ série não atingiram o escore esperado para a identificação das reversões de letras, números e letras dentro das palavras.

A Tabela 2 apresenta o desempenho dos escolares de $1^{\underline{a}}$ série nos subteste do Teste de Desempenho Contínuo. Depois da aplicação do Teste Friedman, verificou-se diferença estatisticamente significante quando comparado o desempenho obtido dos escolares nos subtestes de controle de atenção 1 , controle de atenção 2 , detecção de reversão 1 , detecção de reversão 2 , detecção de reversão 3 e palavras invertidas 1 , evidenciando que entre a execução destas provas os escolares apresentaram diferentes desempenhos.

Como foi verificada diferença estatisticamente significante na Tabela 2, aplicou-se o Teste dos Postos Sinalizados de Wilcoxon para verificar em qual subteste ocorreu melhor desempenho dos escolares. Assim, na Tabela 3, pode-se verificar que ocorreu diferença estatisticamente significante, indicando que os escolares apresentaram melhor desempenho em controle de atenção 1 seguido de controle de atenção 2, detecção de reversão 1, palavras invertidas 1 , detecção de reversão 2 , detecção de reversão 3 (CAO1 > CAO2 > DRO1 > $\mathrm{PIO} 1>\mathrm{DRO} 2>\mathrm{DRO} 3)$, ou seja, que os escolares da $1^{\text {a }}$ série apresentaram desempenho superior para a identificação das letras sem reversão e inversão e de palavras que apresentam uma letra com reversão e inversão do que para a identificação de letras e números isolados.

A Tabela 4 apresenta o desempenho dos escolares de 1aㅡ série nos subtestes do Teste de Memória de Curto Prazo. Depois da aplicação do Teste dos Postos Sinalizados de Wilcoxon, verificouse diferença estatisticamente significante quando comparado o desempenho obtido dos escolares nos subtestes de detecção da reversão e palavras invertidas 2, revelando que entre a execução destas provas os escolares apresentaram diferentes desempenhos, evidenciando que estes escolares identificam, com desempenho superior, a reversão de letras e números isolados do que a inversão de letras dentro da palavra.

$\mathrm{Na}$ Tabela 5, pode-se verificar o desempenho dos escolares de $1^{\underline{a}}$ série nos subtestes do Teste de Controle de Escrita. Depois da aplicação do Teste dos Postos Sinalizados de Wilcoxon, verificou-se que não ocorreu diferença estatisticamente significante quando comparado o desempenho obtido dos escolares nos subtestes de escrita a partir de figuras e cópia, evidenciando desempenho semeIhante entre os escolares.

A Figura 1 apresenta o desempenho dos escolares em relação ao tempo de execução nos subteste do Teste de Desempenho Contínuo. Depois da aplicação do Teste Friedman, verificou-se diferença 
Tabela 1 - Distribuição da média, desvio padrão e valor de $p$ do desempenho obtido e esperado dos escolares na bateria de identificação dos erros de reversão na escrita.

\begin{tabular}{|c|c|c|c|c|}
\hline & Par de Variáveis & Média & $\begin{array}{l}\text { Desvio } \\
\text { Padrão }\end{array}$ & Valor de $p$ \\
\hline \multirow{12}{*}{$\begin{array}{c}\text { Teste de Desempenho } \\
\text { Contínuo }\end{array}$} & CAO1 & 13,33 & 0,92 & \multirow{2}{*}{$0,002^{*}$} \\
\hline & CAE1 & 14,00 & 0,00 & \\
\hline & CAO2 & 7,53 & 2,67 & \multirow{2}{*}{$0,000^{*}$} \\
\hline & CAE2 & 10,00 & 0,00 & \\
\hline & DRO1 & 7,17 & 3,12 & \multirow{2}{*}{$0,000^{*}$} \\
\hline & DRE1 & 10,00 & 0,00 & \\
\hline & DRO2 & 6,40 & 3,47 & \multirow{2}{*}{$0,000^{*}$} \\
\hline & DRE2 & 13,00 & 0,00 & \\
\hline & DRO3 & 5,47 & 4,26 & \multirow{2}{*}{$0,000^{*}$} \\
\hline & DRE3 & 13,00 & 0,00 & \\
\hline & PIO1 & 9,27 & 3,41 & \multirow{2}{*}{$0,000^{*}$} \\
\hline & PIE1 & 16,00 & 0,00 & \\
\hline \multirow{4}{*}{$\begin{array}{l}\text { Teste de memória } \\
\text { de curto prazo }\end{array}$} & DRO4 & 6,20 & 2,92 & \multirow{2}{*}{$0,000^{\star}$} \\
\hline & DRE4 & 10,00 & 0,00 & \\
\hline & $\mathrm{PIO} 2$ & 3,53 & 1,94 & \multirow{2}{*}{$0,000^{*}$} \\
\hline & PIE2 & 8,00 & 0,00 & \\
\hline \multirow{4}{*}{$\begin{array}{l}\text { Teste de Controle } \\
\text { de Escrita }\end{array}$} & EFO & 0,17 & 0,46 & \multirow{2}{*}{$0,000^{\star}$} \\
\hline & EFE & 10,00 & 0,00 & \\
\hline & $\mathrm{CO}$ & 1,73 & 3,91 & \multirow{2}{*}{$0,000^{*}$} \\
\hline & CE & 17,00 & 0,00 & \\
\hline
\end{tabular}

Legenda: CA1: controle de atenção 1, CA2:controle de atenção 2, DR1:detecção de reversão 1, DR2: detecção de reversão 2, DR3: detecção de reversão 3, PI 1:palavras invertidas 1, DR4:detecção da reversão 4, PI2:palavras invertidas 2, EF: escrita a partir de figuras, C: cópia, O: escore obtido, E: escore esperado.

Tabela 2 - Distribuição da média, desvio padrão, valor mínimo, valor máximo, mediana e valor de $p$ dos escolares de $1^{\underline{a}}$ série nos subtestes do Teste de Desempenho Contínuo.

\begin{tabular}{ccccccc}
\hline $\begin{array}{c}\text { Subtestes do Teste de } \\
\text { Desempenho Contínuo }\end{array}$ & Média & $\begin{array}{c}\text { Desvio } \\
\text { Padrão }\end{array}$ & Mínimo & Máximo & Mediana & Valor de p \\
\hline CA1 & 13,33 & 0,92 & 11,00 & 14,00 & 14,00 & \\
CA2 & 7,53 & 2,67 & 1,00 & 10,00 & 8,00 & \\
DR1 & 7,17 & 3,12 & 0,00 & 10,00 & 8,50 & $<0,001^{*}$ \\
DR2 & 6,40 & 3,47 & 0,00 & 13,00 & 7,50 & \\
DR3 & 5,47 & 4,26 & 0,00 & 13,00 & 4,00 & \\
P11 & 9,27 & 3,41 & 0,00 & 15,00 & 9,00 & \\
\hline
\end{tabular}

Legenda: CA1:controle de atenção 1; CA2:controle de atenção 2, DR1:detecção de reversão 1, DR2:detecção de reversão 2, DR3:detecção de reversão 3, PI1:palavras invertidas 1, 
Tabela 3 - Comparação do desempenho dos escolares nos subtestes do Teste de Desempenho Contínuo.

\begin{tabular}{cc}
\hline $\begin{array}{c}\text { Subtestes do Teste de } \\
\text { Desempenho Contínuo }\end{array}$ & Valor de p \\
\hline CA2 X CA1 & $<0,001^{*}$ \\
DR1 X CA1 & $<0,001^{*}$ \\
DR2 X CA1 & $<0,001^{*}$ \\
DR3 X CA1 & $<0,001^{*}$ \\
P11 X CA1 & $<0,001^{*}$ \\
DR1 X CA2 & 0,597 \\
DR2 X CA2 & $0,022^{*}$ \\
DR3 X CA2 & $0,004^{\star}$ \\
PI1 X CA2 & $0,012^{*}$ \\
DR2 X DR1 & 0,055 \\
DR3 X DR1 & $0,016^{\star}$ \\
P11 X DR1 & $0,005^{\star}$ \\
DR3 X DR2 & $0,038^{*}$ \\
PI1 X DR2 & $<0,001^{*}$ \\
PI1 X DR3 & $<0,001^{*}$ \\
\hline
\end{tabular}

Legenda: CA1: controle de atenção 1, CA2:controle de atenção 2 , DR1:detecção de reversão 1, DR2:detecção de reversão 2, DR3:detecção de reversão 3, PI 1:palavras invertidas 1 . estatisticamente significante quando comparado o tempo de realização dos subtestes pelos escolares nos subtestes de controle de atenção 1, controle de atenção 2, detecção de reversão 1, detecção de reversão 2, detecção de reversão 3 e palavras invertidas 1 , evidenciando que no tempo de execução destas provas os escolares apresentaram diferentes desempenhos.

Com a aplicação do Teste dos Postos Sinalizados de Wilcoxon, pode-se verificar na Tabela 6 que ocorreu diferença estatisticamente significante quando comparado o tempo de execução dos subtestes, indicando que os escolares apresentaram desempenho inferior em controle de atenção 1 seguido de controle de atenção 2, detecção de reversão 1 , detecção de reversão 2 , detecção de reversão 3 e palavras invertidas 1 (CAO1 < CAO2 $<$ DRO1 < DRO2 < DRO3 < PIO1), ou seja, que os escolares da 1a série apresentam menor tempo para a identificação de reversão e inversão de letras e números isolados do que em sequência.

$\mathrm{Na}$ Figura 2, pode-se observar o desempenho dos escolares de $1^{\underline{a}}$ série em relação ao tempo de execução nos subtestes do Teste de memória de curta duração e nos subtestes do Teste de Controle de Escrita. Depois da aplicação do Teste dos Postos Sinalizados de Wilcoxon, não se verificou diferença estatisticamente significante quando comparado o tempo de execução nos subtestes de detecção da

Tabela 4 - Distribuição da média, desvio padrão, valor mínimo, valor máximo, mediana e valor de $p$ nos subtestes do Teste de memória de curto prazo.

\begin{tabular}{ccccccc}
\hline $\begin{array}{c}\text { Teste de memória de } \\
\text { curto prazo }\end{array}$ & Média & $\begin{array}{c}\text { Desvio } \\
\text { padrão }\end{array}$ & Mínimo & Máximo & Mediana & Valor de p \\
\hline DR4 & 6,20 & 2,92 & 0,00 & 10,00 & 6,50 & $<0,001^{*}$ \\
PI2 & 3,53 & 1,94 & 0,00 & 7,00 & 4,00 & \\
\hline
\end{tabular}

Legenda: DR4: detecção de reversão 4; PI:palavras invertidas 2.

Tabela 5 - Distribuição da média, desvio padrão, valor mínimo, valor máximo, mediana e valor de $p$ nos subtestes do Teste de Controle de Escrita.

\begin{tabular}{ccccccc}
\hline $\begin{array}{c}\text { Teste de Controle de } \\
\text { Escrita }\end{array}$ & Média & $\begin{array}{c}\text { Desvio } \\
\text { padrão }\end{array}$ & Mínimo & Máximo & Mediana & Valor de $\mathbf{p}$ \\
\hline EF & 0,17 & 0,46 & 0,00 & 2,00 & 0,00 & 0,317 \\
C & 1,73 & 3,91 & 0,00 & 14,00 & 0,00 & \\
\hline
\end{tabular}

Legenda: EF: Escrita a partir de figuras; C: cópia 


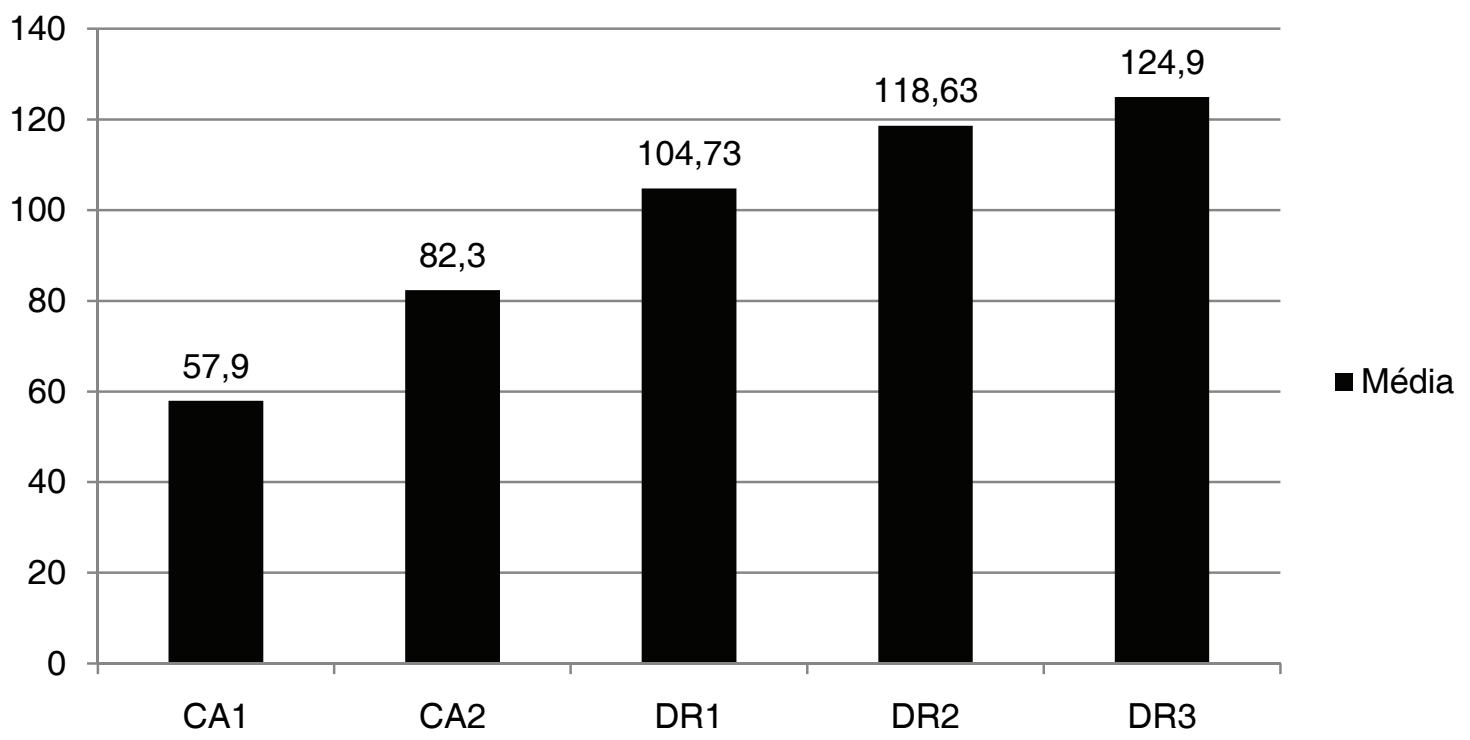

$p=<0,001^{*}$

Legenda: CA1:controle de atenção 1; CA2:controle de atenção 2, DR1:detecção de reversão 1, DR2:detecção de reversão 2 , DR3:detecção de reversão 3, PI1:palavras invertidas 1

Figura 1 - Distribuição da média referente ao tempo de execução dos escolares de 1ª série no Teste de Desempenho Contínuo.

Tabela 6 - Comparação do tempo de execução dos subtestes do Teste de Desempenho Contínuo.

\begin{tabular}{cc}
\hline $\begin{array}{c}\text { Subtestes do Teste de } \\
\text { Desempenho Contínuo }\end{array}$ & Valor de p \\
\hline CA2 X CA1 & $0,002^{*}$ \\
DR1 X CA1 & $<0,001^{*}$ \\
DR2 X CA1 & $<0,001^{*}$ \\
DR3 X CA1 & $<0,001^{*}$ \\
PI1 X CA1 & $<0,001^{*}$ \\
DR1 X CA2 & $0,031^{*}$ \\
DR2 X CA2 & $0,007^{\star}$ \\
DR3 X CA2 & $0,001^{*}$ \\
PI1 X CA2 & $<0,001^{\star}$ \\
DR2 X DR1 & 0,080 \\
DR3 X DR1 & 0,106 \\
PI1 X DR1 & $<0,001^{*}$ \\
DR3 X DR2 & 0,159 \\
PI1 X DR2 & $<0,001^{*}$ \\
PI1 X DR3 & $<0,001^{*}$ \\
\hline
\end{tabular}

Legenda: CAO1: controle de atenção obtido 1; CAO2:controle de atenção obtido 2, DRO1:detecção de reversão obtido 1, DRO2:detecção de reversão obtido 2, , DRO3:detecção de reversão obtido 3, PIO 1:palavras invertidas obtido 1 , reversão e palavras invertidas 2 , evidenciando que o tempo de execução destes subtestes foi semeIhante. Nesta mesma figura verifica-se que ocorreu diferença estatisticamente significante quando comparado o tempo de execução dos escolares nos subtestes de escrita a partir de figuras em relação ao tempo de execução da cópia, evidenciando menor tempo para a realização da cópia.

\section{DISCUSSÃO}

Os resultados deste estudo, de uma forma específica, permitiram conhecer um pouco melhor o processo de análise visual necessário para identificar, codificar e decodificar letras, números e letras em sequência que constituem a palavra, criando, assim, um novo campo de observação para a questão da apropriação da ortografia que não aquela associada à compreensão do princípio alfabético da Língua Portuguesa.

Segundo estudo descrito na literatura nacional ${ }^{27}$, o aprendizado da escrita pressupõe a compreensão de uma série de propriedades ou aspectos da língua escrita que fazem parte do sistema ortográfico. Esse aprendizado engloba: diferenciar o traçado das letras, saber a que sons as letras correspondem, estabelecer correspondências quantitativas, identificar a posição da letra dentro da palavra, compreender que uma mesma letra pode representar vários sons, assim 


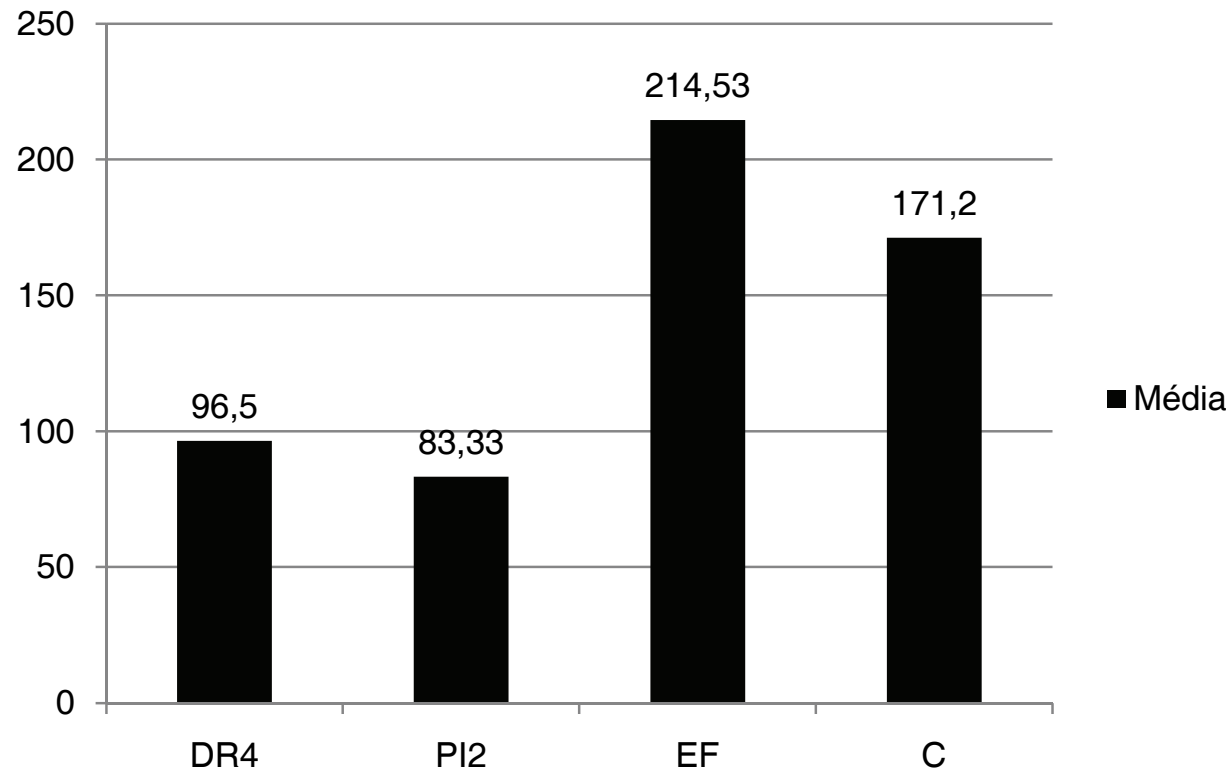

$p=0,144 ; p=0,003^{*}$

Legenda: DR4: detecção de reversão 4; PI2:palavras invertidas 2, EF: escrita a partir de figuras; C: cópia

Figura 2 - Distribuição da média referente ao tempo de execução dos escolares de $1^{\text {a }}$ série nos subtestes de memória de curto prazo e subtestes de controle de escrita.

como um mesmo som pode ser representado por diversas letras.

Neste estudo, foi evidenciado que os escolares da $1^{\underline{a}}$ série apresentaram melhor desempenho na identificação de reversão e inversão de letras dentro de palavras do que isoladas, isto pode ter ocorrido devido aos escolares deste estudo estarem em constante contato com atividades de leitura e escrita, o que favoreceu a representação visual mental das palavras e, portanto, qualquer modificação na estrutura da escrita destas palavras tornase mais fácil de ser identificada, conforme descrito na literatura ${ }^{28}$.

O processo de análise da estrutura da escrita tem seu início com o reconhecimento das palavras e a atribuição de significado, para depois, ser ativada a forma ortográfica das palavras e os processos motores. É a atribuição de significado ao significante. Esse caminho supõe a compreensão do significado do que está escrito e a aferição da ortografia correta. Para escrever uma palavra que Ihe foi ditada, o escolar deverá ter construído a noção de letra, de número, de vogal, de consoante, de palavra e de frase. Além dessas construções, que implicam a construção do sistema de representação e na construção do código, o escolar deverá dominar o sistema de significação, de modo a diferenciar significado e significante ${ }^{1-3,28}$.

Quando solicitada aos escolares a realização de tarefa de memória de curta duração, observou-se que a identificação da reversão para letras e números foi mais facilmente percebida do que a inversão de letras na palavra. Este achado demonstra que quando o escolar se depara com a letra ou o número em reversão, deve-se realizar a identificação do código para ser recuperada a representação visual, o que não necessariamente ocorre quando o escolar está exposto à palavra, pois se a palavra for de alta frequência ocorrerá a liberação do léxico de input visual ${ }^{29}$, que favorecerá o reconhecimento global da palavra e a não-percepção de letras invertidas ou revertidas nas palavras, conforme os resultados encontrados neste estudo.

Em relação ao teste de controle de escrita, os escolares apresentaram desempenhos semeIhantes para escrita de palavras e realização de cópia. Apesar de a ortografia da maior parte das palavras escritas do procedimento utilizado não ter sido apresentada de forma correta, a produção das letras sem a possível reversão e inversão dentro das palavras pelos escolares foi correta. Entretanto, na cópia, os escolares não copiaram a inversão, escrevendo a letra da forma correta. Esses achados podem ser explicados pelo fato de estes escolares já apresentarem em seu léxico mental a representação visual da letra justificando a não realização de cópia das letras invertidas e a escrita das palavras sem letras revertidas ou invertidas, conforme descrito na literatura ${ }^{30}$. 
Quanto ao tempo de realização dos subtestes, verificou-se que os escolares deste estudo apresentaram menor tempo para a identificação de reversão e inversão de letras e números isolados do que em sequência, demonstrando que a diferenciação do traçado das letras, o estabelecimento de correspondências quantitativas e identificação da posição da letra dentro da palavra é parte do aprendizado da escrita que os escolares vão realizar ao longo da alfabetização ${ }^{27}$.

Em decorrência do exposto, como o escolar deste estudo está em fase de apropriação do sistema de escrita, a habilidade de cópia foi realizada em menor tempo do que a escrita a partir da figura, demonstrando que o escolar nesta fase está mais perceptivo e susceptível aos detalhes da formação da letra ou números, o que acarreta a lentidão ou identificação no processo da escrita das palavras.

Os resultados deste estudo apontam para a necessidade de ampliação das pesquisas na área fonoaudiológica, pois a ocorrência de inversões tem sido descrita na literatura como a alteração ortográfica menos frequente ${ }^{31-34}$. Entretanto, como no Brasil ainda são restritos os protocolos específicos para a investigação destes tipos de erros, bem como os protocolos de investigação sobre a relação percepto-viso-motora e a aquisição da leitura e escrita, pode-se considerar que ainda é precoce essa afirmação, uma vez que este estudo revelou algumas dificuldades quanto à identificação dos erros de inversão e reversão, que devem ser monitoradas ao longo da aprendizagem da leitura e escrita.

\section{CONCLUSÃO}

Com base nos resultados apresentados nesse estudo conclui-se que os escolares de $1^{\underline{a}}$ série deste estudo apresentaram erros de inversão e reversão de letras e números isolados e em seqüência, que neste momento da alfabetização podem ser considerados como erros de construção ou de apropriação do sistema ortográfico.

Desta forma, os dados preliminares deste estudo alerta para a necessidade de continuidade de pesquisas que utilizem esta Bateria como procedimento de identificação dos erros de reversão e de inversão na população escolar de diferentes séries, pois somente assim, será possível determinar se as características de reversão e de inversão de letras e números constituem o quadro de dislexia ou distúrbio de aprendizagem, pois a partir do conhecimento do desenvolvimento típico em leitura e escrita é que a determinação do desenvolvimento atípico dos escolares que apresentam tais transtornos de aprendizagem ocorrerá de forma fidedigna e precisa.

\begin{abstract}
Purpose: to characterize the performance of students from $1^{\text {st }}$ grade in the inversion and reversion errors in the written identification Brazilian Test. Methods: 30 students from $1^{\text {st }}$ grade of a public school in Marília City, from both genders, ranging from 7 to 7 years and 11 month old took part. As a procedure, we carried out the inversion and reversion errors in the written identification Test Brazilian adaptation. This Test is composed of 3 subtests: continuum performance subtest, short term memory subtest and written control subtest. Each subtest is composed of a test aiming to identify the skill of the student in identifying the inversion and reversion of isolated letters and numbers and sequence of letters and numbers. Results: the results revealed that the students from $1^{\text {st }}$ grade submitted superior performance in order to identify the reversion and inversion of letters inside the words than isolated ones, superior performance to identify the reversion to letters and numbers compared to the inversion of letters in the word. Moreover, the students of this study submitted smaller time frames in order to identify the reversion and inversion of isolated letters and numbers than in sequence. Conclusion: the Test adapted to this study allowed us to know the profile of $1^{\text {st }}$ grade students in order to identify the inversion and reversion errors, however, it is necessary to apply it again in order to verify the occurrence of reversion and inversion errors identification in populations of different literacy phases.
\end{abstract}

KEYWORDS: Evaluation; Handwriting; Learning 


\section{REFERÊNCIAS}

1. Capellini AS, Souza AV. Avaliação da função motora fina, sensorial e perceptiva em escolares com dislexia. In: Sennyey AL, Capovilla FC, Montiel, JM. Transtornos da aprendizagem da avaliação à reabilitação. São Paulo: Artes Médicas; 2008. p. 55-64.

2. Schirmer CR, Fontoura DR, Nunes ML. Distúrbios da aquisição da linguagem e da aprendizagem. J Pediatr. 2004; 80(suppl 2):95-103 .

3. Kolb B, Whishaw IQ. Neurociências do comportamento. Barueri: Manole; 2002.

4. Mathes PG, Denton CA. The prevention and identification of reading disabilities. Semin Pediatr Neurol. 2002; 9(3):185-91.

5. Boets B, Wouters J, Van Wieringen A, De Smedt B, Ghesquiére $P$. Modelling relations between sensory processing, speech perception, orthographic and phonological ability, and literacy achievement. Brain Lang. 2008; 106(1):29-40.

6. Salles JF, Parente MAMP. Avaliação da leitura e escrita de palavras em crianças de $2^{a}$ série: abordagem neuropsicológica cognitiva. Psicol Reflex Crit. 2007; 20(2):220-8.

7. Mousinho R, Correa J. Habilidades linguístico-cognitivos em leitores e não-leitores. Pró-Fono. 2009; 21(2):113-8.

8. Stivanin L, Scheuer C. Comparação do tempo de latência entre nomeação e leitura em escolares. Psicol Estud. 2008; 13(1):89-96.

9. Smythe I, Everatt J, Al-Menaye N, He X, Capellini SA, Gyarmathy E, Siegel LS Predictors of word-level literacy amongst grade 3 children in five diverse languages. Dyslexia. 2008; 14(3):170-87.

10. Swanson HL, Howard CB, Saez L. Do different components of working memory underlie different subgroups of reading disabilities? J Learn Disab. 2006; 39(3):252-69.

11. Chard DJ, Stoolmiller M, Harn BA, Wanzek J, Vaughn S, Linan-Thompson S, et al. Predicting reading success in a multilevel schoolwide reading model. J Learn Disab. 2008; 41(2):174-88.

12. Harn, BA, Stoolmiller, M, Chard, DJ. Measuring the dimensions of alphabetic principle on the reading development of first graders. The role of automaticity and unitization. J Learn Disab. 2008; 41(2):143-57.

13. Kirk C, Gillon GT. Integrated morphological awareness intervention as a tool for improving literacy. Lang Speech Hear Serv Sch. 2009; 40(3):341-51.
14. Bolger DJ, Minas J, Burman DD, Booth JR. Differential effects of orthographic and phonological consistency in cortex for children with and without reading impairment. Neuropsychol. 2008; 46(14):3210-24.

15. Monteiro DC, Nordi C, Silva CRG, Doricci GP, Marrara JL. Que tipos de erros ortográficos ainda existem na $5^{\text {a }}$ série do ensino fundamental? Fono Atual. 2004; 29:40-9.

16. Ávila CRB, Ramos CS, Frigerio MC, Lucas $\mathrm{S}$. Análise da escrita de escolares de $4^{\underline{a}}$ série fundamental das redes pública e particular. Rev Soc Bras Fonoaudiol. 2001; 1:23-8.

17. Cunha VLO, Capellini SA. Desempenho de escolares da 1a a $4 a$ série do ensino fundamental nas provas de habilidades metafonológicas e de leitura - PROHMELE. Rev Soc Bras Fonoaudiol. 2009; 14(1):56-68.

18. Gray A, Mccutchen D. Young readers' use of phonological information: phonological awareness, memory, and comprehension. J Learn Disabil. 2006; 39(4):325-33.

19. Moojen S, Lamprecht R, Santos RM, Freitas GM, Brodacz R, Siqueira M, et al. CONFIAS Consciência Fonológica: Instrumento de Avaliação Sequencial. São Paulo: Casa do Psicólogo; 2003.

20. Cárnio MS, Stivanin, L, Vieira, MP, Amaro, L, Martins, VO, Carvalho, E et al. Habilidades de consciência fonológica e letramento em crianças do ensino fundamental. Rev Soc Bras Fonoaudiol. 2006; 11(4):231-42.

21. Gindri G, Keske-Soares M, Mota HB. Memória de trabalho, consciência fonológica e hipótese de escrita. Pró-Fono. 2007; 19(3):313-22.

22. Zorzi JL. Aprender a escrever: a apropriação do sistema ortográfico. Porto Alegre: Artmed; 1998.

23. Bartholomeu D, Sisto FF, Rueda FJM. Dificuldades de aprendizagem na escrita e características emocionais de crianças. Psicol Estud. 2006; 11(1):139-46.

24. Wolter JA, Wood A, D'zatko KW. The influence of morphological awareness on the literacy development of first-grade children. Lang Speech Hear Serv Sch. 2009; 40(3):286-98.

25. Ferretti G, Mazzotti S, Brizzolara D. Visual scanning and reading ability in normal and dyslexic children. Behav Neurol. 2008; 19(1-2):87-92.

26. Terepocki M, Kruk RS, Willown DM. The incidence and nature of letter orientation erros in reading disability. J Learn Disab. 2002; 35(3):214-33. 27. Dias RS, Avila, CRB. Uso e conhecimento ortográfico no transtorno específico de leitura. Rev Soc Bras Fonoaudiol. 2008; 13(4):381-90.

28. Kingeski MF, Sisto FF. Reconhecimento de palavras nas séries iniciais do ensino fundamental. Rev Teor Prat Educ. 2004; 7(2):183-90. 
29. Pinheiro AMV, Lúcio OS, Silva DMR. Avaliação cognitiva de leitura: o efeito de regularidade grafema-fonema e fonema-grafema na leitura em voz alta de palavras isoladas no português do Brasil. Psicol Teor Pratic. 2008; 10(2):16-30.

30. Zorzi JL. A inversão de letras na escrita: o fantasma do espelhamento. Pró-Fono. 2001; 13(2):212-8.

31. Zucoloto KA, Sisto FF. Dificuldades de aprendizagem em escrita e compreensão em leitura. Inter Psicol. 2002; 6(2):157-66.
32. Vieira RMR, Grosso PMP, Zorzi JL, Chiappetta ALML. Estudos comparativos sobre a influência da leitura nos erros da escrita entre meninos e meninas de quarta série. Rev. CEFAC. 2006; 8(3):281-8.

33. Zorzi JL. As inversões de letras na escrita, o fantasma do espelhamento. Psicopedagogia. 2001; 13(2):212-8.

34. Zorzi JL, Ciasca SM. Caracterização dos erros ortográficos em crianças com transtornos de aprendizagem. Rev. CEFAC. 2008; 10(3):321-31.

DOI: 10.1590/S1516-18462010005000060

RECEBIDO EM: 18/08/2009

ACEITO EM: 09/02/2010

Endereço para correspondência:

Simone Aparecida Capellini

Rua Bartolomeu de Gusmão, 10-84

Bauru - SP

CEP: 17017-336

E-mail: sacap@uol.com.br 